\title{
Modern markers for evaluating bone disease in multiple myeloma (Review)
}

\author{
VLAD POP $^{1,2}$, ANDRADA PARVU ${ }^{1,2}$, ALEXANDRA CRACIUN $^{3}$, \\ ANCA DANIELA FARCAS ${ }^{4,5}$, GHEORGHE TOMOAIA ${ }^{6}$ and ANCA BOJAN ${ }^{1,2}$ \\ ${ }^{1}$ Hematology Department, 'Iuliu Haţieganu' University of Medicine and Pharmacy; \\ ${ }^{2}$ Hematology Department, 'Prof. Dr. Ioan Chiricuta' Oncological Institute, 400015 Cluj-Napoca; \\ ${ }^{3}$ Medical Biochemistry Department, 'Iuliu Haţieganu' University of Medicine and Pharmacy, \\ 400012 Cluj-Napoca; ${ }^{4}$ Internal Medicine Department, 'Iuliu Haţieganu' University of \\ Medicine and Pharmacy; ${ }^{5}$ Cardiology Department, Emergency County Clinic Hospital, \\ 400006 Cluj-Napoca; ${ }^{6}$ Orthopedics and Traumatology Department, \\ 'Iuliu Haţieganu' University of Medicine and Pharmacy, 400132 Cluj-Napoca, Romania
}

Received July 21, 2021; Accepted August 20, 2021

DOI: $10.3892 / \mathrm{etm} .2021 .10764$

\begin{abstract}
Multiple myeloma (MM) is a bone marrow neoplasia with increasing incidence compared to previous years. Although new therapeutic molecules have been introduced, it remains an incurable disease with severe repercussions to patients. For many patients, bone disease represents a severe problem often causing pain, pathological bone fractures, and spinal cord compression, which affects the quality of life. This article analyzes the main markers of bone destruction in MM as well as risk factors for severe bone damage. Bone complications have a negative impact on the quality of life of patients with MM, along with other associated complications (renal failure, hypogammaglobulinemia, osteolytic bone disease, hypercalcemia, anemia). The markers of bone destruction described in this article include: interleukin (IL)-6, tumor necrosis factor (TNF)- $\alpha$, receptor activator of nuclear factor kappa-B ligand (RANKL), osteoprotegerin (OPG), amino- and
\end{abstract}

Correspondence to: Dr Andrada Parvu, Hematology Department, 'Iuliu Haţieganu' University of Medicine and Pharmacy, 34-36 Republicii Street, 400015 Cluj-Napoca, Romania

E-mail: parvuandrada@hotmail.com

Abbreviations: IL-6, interleukin-6; RANKL, receptor activator of nuclear factor kappa-B ligand; TNF- $\alpha$, tumor necrosis factor- $\alpha$; $\beta$-CTx, $\beta$-crosslaps; OPG, osteoprotegerin; ALP, alkaline phosphatase; MM, multiple myeloma; BSP, human bone sialoprotein; DKK1, dickkopf-1 secreted glycoprotein; BTMs, bone turnover markers; BAP, bone-specific alkaline phosphatase; PINP, type I procollagen amino terminal propeptide; CTX-1, type I C-terminal cross-linking telopeptide; NTX-1, type $1 \mathrm{~N}$-terminal cross-linking telopeptide; MBD, myeloma bone disease; MGUS, monoclonal gammopathy of undetermined significance

Key words: IL-6, RANKL, TNF- $\alpha, \beta-\mathrm{CTx}$, ALP, multiple myeloma carboxy-terminal cross-linking telopeptide of type I collagen (NTX, CTX), human bone sialoprotein (BSP) and dickkopf-1 secreted glycoprotein (DKK1). The future practical applicability of this literature review would be the large-scale determination of markers of bone destruction that correlate with the negative evolution to complications of bone disease or the implications that these markers have in regards to treatment.

\section{Contents}

1. Introduction

2. Physiopathology of bone lesions in multiple myeloma (MM)

3. Cytokine profiles

4. $\beta$-crosslaps $(\beta$-CTx $)$

5. Osteoprotegerin (OPG)

6. Amino- and carboxy-terminal cross-linking telopeptide of type I collagen

7. Human bone sialoprotein (BSP)

8. Dickkopf-1 secreted glycoprotein (DKK1)

9. Conclusions

\section{Introduction}

Myeloma is a disease characterized by clonal expansion of malignant plasma cells accumulated in the marrow leading to cytopenia, hypogammaglobulinemia, osteolytic bone disease, hypercalcemia, and renal dysfunction (1). The incidence of multiple myeloma (MM) accounts for $1 \%$ of all cancers and $10 \%$ of all hematological malignancies, with an incidence in Europe of 4.5-6.0/100,000/year; the median age at diagnosis being 72 years, and with a mortality of 4.1/100,000/year (2). Some patients with MM emerge from an asymptomatic pre-malignant stage, monoclonal gammopathy of undetermined significance (MGUS), which has a rate of progression 
to $\mathrm{MM}$ of $1 \%$ per year, comparative smoldering or indolent MM (asymptomatic but more advanced pre-malignant stage) which has a rate of progression of $10 \%$ per year during the first five years following diagnosis (3).

Evidence of end-organ damage manifested by anemia, lytic bone lesions, hypercalcemia, renal insufficiency, amyloidosis, hyperviscosity, and recurrent infections, is suggestive of symptomatic myeloma. The diagnostic criteria for myeloma consist of evidence of either $10 \%$ or more of clonal bone marrow plasma cells of biopsy-proven bony or extramedullary plasmacytoma and the presence of one or more myeloma defining events [the CRAB criteria (hypercalcemia, renal failure, anemia, lytic bone lesions)], and three specific biomarkers of malignancy (clonal bone marrow plasma cells either $60 \%$ or more, the serum-free light chain of 100 or higher and at least one focal lesions on MRI studies) (4).

One of the active myeloma criteria of bone lesions can often lead to osteopenia and bone fractures in the pathological bone $(4,5)$. The osteolytic bone disease results from an increased osteoclast activity and reduced osteoblast function, characteristic of myeloma. Bone loss in MM is multifactorial; during the disease most of the patients develop a severe osteolytic bone disease $(6,7)$. It is still unclear why bone destruction is a common component of this disease.

Myeloma cells secrete pathological monoclonal immunoglobulins, and the presence of monoclonal immunoglobulins in serum is a measure of tumor burden $(5,8)$.

For many patients, the bone disease is a serious concern, often causing pain, pathological bone fractures with different localization producing specific complications. Typical examples are vertebral osteolysis which may produce pathological fractures and spinal collapse with medullar compression and different neurological symptoms. Considering all the clinical consequences of MM (renal insufficiency, hyperviscosity, anemia), the bone disease in MM has the most significant impact on patient quality of life $(8,9)$. An important aspect of approaching MM is studying bone disease markers. It is important to define serum risk factors for quantifying bone lesions and risk factors predicting future severe bone lesions.

\section{Physiopathology of bone lesions in multiple myeloma (MM)}

The bone disease occurs through several mechanisms that cause an imbalance in bone remodeling, so bone resorption increases compared to the bone formation that is suppressed. Changes in bone modeling occur due to the secretion of factors that have the direct ability to modulate osteoclasts or osteoblasts $(5,10,11)$.

The bone marrow microenvironment is composed of endothelial, stromal, and immune cells, as well as cytokines and participates in both the pathogenesis and progression of MM (12). The bone structure is normally made up of a mineralized and organic part of collagen and a portion made up of non-collagenous proteins. In normal physiological states, homeostasis is maintained through osteocytes, osteoclasts and osteoblasts, which have the role of balancing bone formation and bone resorption $(6,13)$. Osteocytes represent $90-95 \%$ of all bone cells, and osteoclasts and osteoblasts less than $10 \%$. Osteoblasts are mononuclear cells that contain the enzyme alkaline phosphatase (ALP), used as a marker of osteoblastic activity (6).

An alternative for assessing bone condition in MM includes bone turnover markers (BTMs). These biochemical markers of bone fluctuation provide information on bone degradation and formation. They could have a predictive role in the bone condition of patients diagnosed with myeloma $(5,10)$.

BTMs are products of osteoblast activity and include bone-specific alkaline phosphatase (BAP), representing membrane-bound osteoblast enzymes produced during bone formation (5).

In percentage terms, BAP represents about half of the total ALP in healthy subjects. Compared to ALP, BAP is a more sensitive method that reflects both bone formation and bone degradation. Serum BAP provides a marked correlation with the dynamic parameters of bone formation (14). Statistically significant correlations between BAP levels and bone pain, lytic lesions, and bone fractures have been described in numerous studies on MM $(10,14,15)$.

\section{Cytokine profiles}

Numerous studies have been performed focusing on the exploration of serum cytokine levels in patients diagnosed with MM, and have found that their high serum levels are correlated with aggressive or symptomatic disease (16). Other studies have identified prognostic factors such as genetic or epigenetic abnormalities, and anomalies of the immune system that could predict the evolution of patients with myeloma. Recently, although current therapies have improved MM patient prognosis, MM still remains an incurable disease (12).

Not all cytokines participate in the pathogenesis, progression or have a role in the prognosis of MM. The most important cytokines include: IL-6, RANKL, TNF- $\alpha$, and $\beta$-crosslaps $(\beta-\mathrm{CTx})(12,16)$.

$I L-6$. The growth, division and survival of malignant plasma cells are supported by IL-6, which plays an important role in the pathophysiology of MM. It can infiltrate tumors and tumor cells themselves as well as fibroblast stromal cells. It belongs to the interleukin family and stimulates the inflammatory and autoimmune processes in many diseases $(17,18)$.

IL-6 has a role in stimulating the humoral and cellular immune response by acting on both $\mathrm{B}$ lymphocytes and T lymphocytes (19). It also acts as a factor of growth and differentiation of $\mathrm{B}$ cells and stimulates their immunoglobulin production. IL-6 is involved in the pathogenesis of myeloma, and it is used as a prognostic factor for the disease $(16,20-22)$.

Different cytokines take part in the pathogenesis, progression, and prognosis of MM. This was demonstrated in a retrospective study by $\mathrm{Gu}$ et $\mathrm{al}$, who aimed to investigate the correlations between serum cytokine levels and clinical symptoms. An increase in IL-6 levels was observed in newly diagnosed patients with MM compared to healthy subjects, indicating that IL-6 may be a strong predictor for prognosis of MM (12).

Trabecular and endochondral bone loss was found to be significantly improved in the presence of myelomatous cells, being associated with osteoclast differentiation (23). 
TNF- $\alpha$. TNF- $\alpha$ is a cytokine with an important role in the immune system and belongs to the TNF superfamily. This cytokine has an important role in many cellular pathways, mainly in cellular proliferation, differentiation, survival, and cell death (24-26).

Many factors such as osmotic stress with ultraviolet light, various chemical agents, and viruses, can stimulate the production of TNF- $\alpha$. In addition, fibrin can induce the production of TNF- $\alpha$ in macrophages. Initially TNF- $\alpha$ is a type II transmembrane homotrimer protein that can undergo a cleavage process by metalloprotease. Subsequently this homotrimer protein can be released as a polypeptide. Of these polypeptides, only three can polymerize and produce circulating TNF- $\alpha$ with a characteristic pyramid shape. Due to the receptor binding sites, located under these structures, it gives them the ability to attach to receptor molecules (24).

There are two receptors of TNF- $\alpha$ : tumor necrosis factor 1 receptor (TNFR1), present on almost all cell types, while tumor necrosis factor 2 receptor (TNFR2) expression is restricted to minor subpopulations of the lymphoid system including regulatory T cells (Tregs) and myeloid suppressor cells, endothelial cells, and certain tumor cells $(23,27)$.

TNF- $\alpha$ has fundamental roles in the normal immune system and is a proliferative factor for malignant plasma cells (15). It is one of the three major proinflammatory cytokines besides IL-1 and IL-6 and works synergistically with IL-6 and RANKL; therefore, it regulates proinflammatory responses, neovascularization and induces osteoclastogenesis $(16,24)$.

Aberrant expression of TNFR2 on tumor cells has been shown in many recent studies with evidence of the key role of this receptor in tumor progression in rodents and humans (27). TNFR2 is aberrantly expressed on various cancer cells and highly immunosuppressive Tregs accumulated in the tumor microenvironment. Tregs promote cancer cell survival and tumor growth. TNFR2 is considered to be a prospective target for cancer immunotherapy.

The presence of TNF- $\alpha$ polymorphisms has been associated with an increased risk of MM, as presented by Hong et al (28) in the meta-analysis. TNF- $\alpha 308$ polymorphism has been rarely observed in patients with myeloma; however, it may have a role in the pathogenesis of MM. In an attempt to find links between TNF- $\alpha$ polymorphisms in predicting MM risk, this meta-analysis was performed in many geographic regions. The heterogeneity of the group can explain the genetic diversity caused by daily living habits, environmental differences, the level of economic development, but also by the sex and age of the people enrolled in the study. The results showed that the $\mathrm{TNF} \alpha-857$ gene had significant associations with MM risk (28).

Lenalidomide and pomalidomide are analogues of thalidomide known as immunomodulatory drugs (IMiDs) that have an anti-angiogenic, anti-inflammatory antitumor necrosis factor activity in monocytes. Immunomodulators have the opposite effect on T cells, which increase TNF- $\alpha$ production. IMiDs have a direct effect on the proliferative capacity of myeloma cells (29).

$R A N K L$. A member of the TNF- $\alpha$ superfamily, RANK is a transmembrane signaling receptor found predominantly on the surface of osteoclasts. RANK is produced by bone marrow stromal cells, osteoblasts and T lymphocytes $(6,30)$.
The RANKL is secreted mainly by osteocytes, bone marrow-derived stem cells (BMSCs) and osteoblasts. RANKL induces differentiation into mature cells by binding RANK to immature osteoclasts. A positive correlation was noted between the incidence of osteolytic lesions and increased serum RANKL $(30,31)$.

Excessive RANKL production has been shown to be correlated with increased bone resorption. Inhibition of RANKL in patients diagnosed with MM could prevent bone destruction $(31,32)$. A possible treatment with an anti-RANKL monoclonal antibody, a treatment that could cause its inhibition, has been the aim of clinical studies $(31,33)$.

Denosumab is a fully human monoclonal antibody, administered subcutaneously that can neutralize RANKL thus preventing the interaction of RANKL with RANK. This monoclonal antibody binds only to RANKL providing high affinity and specificity (31). In a randomized, double-blind, phase 3 study compared to zoledronic acid, denosumab was shown to be superior in preventing skeletal-related events. In the denosumab arm, a progression-free survival (PFS) advantage was observed; yet, further more complex investigations are needed to confirm this effect $(34,35)$.

\section{4. $\beta$-crosslaps $(\beta-\mathrm{CTx})$}

Bone markers can be used to monitor therapy (including treatment compliance) and to estimate the risk of fractures caused by osteoporosis. The usefulness of bone markers is not limited to the evaluation of osteoporosis, as they have clinical value in bone diseases, such as MM.

In an increased proportion of $>90 \%$, the bone organic matrix is composed of type I collagen, which subsequently follows a degradation process. In the case of increased bone resorption, there is an increased degradation of type I collagen, with increasing levels of fragments released into the circulation $(11,36)$. The $\alpha$-aspartic acid present in the $\mathrm{C}$-terminal telopeptides is converted to the $\beta$ ( $\beta$-CTx) form. These isomerized telopeptides are specific for the degradation of type I collagen that predominates in the bone. $\beta$-CTx is a specific and stable marker of bone resorption, expressed in the early stages of type I collagen degradation $(11,36,37)$.

The degree of imbalance between bone resorption and bone formation can be expressed by the ratio of $\beta$-CTx/PINP (type I procollagen amino terminal propeptide). Studies have shown that CTx and PINP are important parameters values for clinical evaluation of osteoclast and osteoblast activity (38).

PINP and $\beta$-CTx markers are widely used due to their specificity for evaluating bone turnover in the clinical setting, low analytical variability, and satisfactory results in clinical trials (39).

Markers reflecting bone degradation, including $\beta-\mathrm{CTx}$, are significantly higher in patients with MM than in patients with MGUS. Furthermore, these markers can determine the degree of bone damage and disease progression.

Thereby, the clinical significance of PINP and $\beta$-CTx was investigated in patients with MM or MGUS. A cohort study of 241 patients showed increased serum levels of PINP and $\beta$-CTx in patients with MM than in patients with MGUS (40).

The serum levels of $\beta$-CTx represent a sensitive marker capable of detecting the degree of bone resorption in MM. 
This marker has the advantage of predicting subclinical bone disease before the onset of manifested lytic lesions $(40,41)$. In the management of metastatic bone diseases, $\beta$-CTx looks promising for detecting bone metastases, evaluating prognosis, and monitoring treatment $(11,36)$.

\section{Osteoprotegerin (OPG)}

OPG, an inhibitor of RANKL, is a receptor responsible for increased osteoclastogenesis and osteoclast activity. OPG is secreted by osteoblasts, blocks the function of osteoclasts and maintains the balance of bone formation and resorption. OPG and other protagonists regulate the binding of RANKL or RANK, which are regulated by other signaling factors. In the myeloma bone marrow microenvironment, the RANKL/OPG ratio is in favor of RANKL, leading to increased osteoclastogenesis and increased bone resorption $(42,43)$.

Previous research has shown that in MM, the soluble RANKL/OPG ratio is increased, while serum OPG levels are low. It has also been found that the administration of zoledronic acid reduce the RANKL/OPG ratio by increasing OPG serum level (42). Raje et al presented in a review that lenalidomide and pomalidomide, both immunomodulatory drugs, inhibit the interaction between osteoprogenitor cells and myeloma, and may prevent an imbalance in the RANKL/OPG ratio (43).

\section{Amino- and carboxy-terminal cross-linking telopeptide of type I collagen}

In myeloma bone disease (MBD), an increased RANKL production in the bone microenvironment of MM, alters the RANKL/OPG ratio, leading to increased collagen degradation in the osteoclastic-mediated bone matrix. Bone resorption markers obtained from type I collagen degradation are type I C-terminal cross-linking telopeptide (CTX-1) and type 1 $\mathrm{N}$-terminal cross-linking telopeptide (NTX-1) $(38,42)$.

Biochemical markers of NTX, CTX, or CTX bone resorption generated by matrix metalloproteinases (ICTP) provide bone dynamic information and reflect the activity of the bone disease. These markers can also assess the degree of bone disease, can evaluate the response to treatment and the risk of skeletal morbidity in patients with MM (42).

Bone resorption markers NTX, ICTP, and CTX, are increasingly used, replacing the old methods of bone disease diagnostics, mainly due to their present characteristics.

CTX-1 is an inexpensive method used for monitoring patients with MM, is robust and reproductible, with potential for bone recurrence detection.

Ting et al presented in a research paper, a significantly elevated level of CTX-1 in patients with lytic lesions, whose MRI showed no bone marrow abnormalities or lytic bone destruction. The study conclusions highlight the important role of CTX-1 in predicting subclinical bone disease before lithic bone destruction occurs (38).

\section{Human bone sialoprotein (BSP)}

BSP is a major non-collagenous extracellular protein of mineralized tissues. BSP is produced during bone morphogenesis by osteoblasts, osteoclasts, osteocytes, and hypertrophic chondrocytes.

One particular interest in malignant bone disease is the serum levels of BSP, which were found to differ depending on the stage of the bone disease, being high in patients with MM. Additionally, Maaroufi et al showed in an article that BSP serum levels play an important role in early diagnosis and treatment of patients with MM. Patients included in the study with normal serum BSP levels survived longer than patients with high serum values, showing that BSP levels are correlated with bone marrow plasma cell content $(44,45)$.

\section{Dickkopf-1 secreted glycoprotein (DKK1)}

DKK1 is an inhibitor of the Wnt signaling pathway, is generally expressed in myeloma cells, and is restricted in normal tissues. DKK1 actively participates in the regulation of MBD by inhibiting osteoblasts and activating osteoclasts.

Reducing osteolytic bone resorption and increasing bone formation can be achieved by blocking DKK1 activity. For this reason, new therapies have appeared; anti-DKK1 treatments that can control the development and progression of MM. Elevated serum levels of DKK1 have been shown to cause osteolytic damage, predominantly in plasma cell myeloma (PCM). In a review by Zhou et al decreased DKK1 levels were found in patients with MM who achieved complete or partial remission. The results of the study clearly demonstrate that myelomatous cells are the main source of circulating DKK1 proteins. In the future, new clinical trials with anti-DKK1 therapies should be performed (46).

\section{Conclusions}

In recent years, the emergence of new therapeutic molecules has led to the increased survival of myeloma patients, but it continues to be an incurable disease. Bone disease in MM patients represents a serious problem leading to morbidities and mortality.

Excessive osteoclastic bone resorption and decreased osteoblastic bone formation are responsible for bone loss subsequently associated with bone pain. The quality of life of patients with MM can be affected by the appearance of these complications during the disease with clinical consequences, including pathological bone fractures and sometimes vertebral collapse.

Bone lesion markers in MM can be used as: markers of prognostic factors (IL-6), therapeutic targets (anti-IL-6 agents), and for treatment monitoring ( $\beta$-CTx).

Further studies are needed to understand the etiology and the pathology of the disease and particularly of the bone disease associated with MM, and to define new prognostic factors of bone lesions. An important future issue is to discover, define and study therapeutic targets for bone lesions.

\section{Acknowledgements}

Not applicable.

\section{Funding}

No funding was received. 


\section{Availability of data and materials}

All information included in this review is documented by relevant references.

\section{Authors' contributions}

VP, AP and AB contributed substantially to the conception and design of the study, the acquisition, selection, analysis, and interpretation of the data, and were involved in the drafting of the manuscript. AC, ADF, GT contributed substantially to the acquisition, analysis and interpretation of the data and were involved in the drafting of the manuscript. All authors contributed to the revision of the manuscript. VP, AP, AB, and GT had an equal contribution in regards to this article. All authors read and approved the final manuscript for publication.

\section{Ethics approval and consent to participate}

Not applicable.

\section{Patient consent for publication}

Not applicable.

\section{Competing interests}

The authors declare that they have no competing interests.

\section{References}

1. Mohan M, Kumar M, Samant R, Van Hemert R Jr, Tian E, Desai S, van Rhee F, Thanendrarajan S, Schinke C, Suva LJ, et al: Bone remineralization of lytic lesions in multiple myeloma-the Arkansas experience. Bone 146: 115876, 2021.

2. Martino A, Buda G, Maggini V, Lapi F, Lupia A, Di Bello D, Orciuolo E, Galimberti S, Barale R, Petrini M and Rossi AM Could age modify the effect of genetic variants in IL6 and TNF- $\alpha$ genes in multiple myeloma? Leuk Res 36: 594-597, 2012.

3. Clay-Gilmour AI, Hildebrandt MAT, Brown EE, Hofmann JN, Spinelli JJ, Giles GG, Cozen W, Bhatti P, Wu X, Waller RG, et al Coinherited genetics of multiple myeloma and its precursor monoclonal gammopathy of undetermined significance. Blood Adv 4: 2789-2797, 2020.

4. Gaudio A, Xourafa A, Rapisarda R, Zanoli L, Signorelli SS and Castellino P: Hematological diseases and osteoporosis. Int J Mol Sci 21: 3538, 2020.

5. Auzina D, Erts R and Lejniece S: Prognostic value of the bone turnover markers in multiple myeloma. Exp Oncol 39: 53-56, 2017

6. Terpos E, Christoulas D and Gavriatopoulou M: Biology and treatment of myeloma related bone disease. Metabolism 80: 80-90, 2018

7. Łacina P, Butrym A, Humiński M, Dratwa M, Frontkiewicz D, Mazur G and Bogunia-Kubik K: Association of RANK and RANKL gene polymorphism with survival and calcium levels in multiple myeloma. Mol Carcinog 60: 106-112, 2021.

8. Westhrin M, Kovcic V, Zhang Z, Moen SH, Nedal TMV, Bondt A, Holst S, Misund K, Buene G, Sundan A, et al: Monoclonal immunoglobulins promote bone loss in multiple myeloma. Blood 136 2656-2666, 2020.

9. Tanimoto K, Hiasa M, Tenshin H, Teramachi J, Oda A, Harada T, Ashtar M, Sogabe K, Oura M, Endo I, et al: Mechanical unloading enhances bone destruction and tumor expansion in multiple myeloma: Critical roles of osteocytic RANKL induction. Bone Rep 13: 100425, 2020.

10. Annibali O, Petrucci MT, Santini D, Bongarzoni V, Russano M, Pisani F, Venditti O, Pantano F, Rago A, Siniscalchi A, et al: Alkaline phosphatase (ALP) levels in multiple myeloma and solid cancers with bone lesions: Is there any difference? J Bone Oncol 26: 100338, 2021.
11. Diaz-delCastillo M, Chantry AD, Lawson MA and Heegaard AM: Multiple myeloma-a painful disease of the bone marrow. Semin Cell Dev Biol 12: 49-58, 2021.

12. Gu J, Huang X, Zhang Y, Bao C, Zhou Z and Jin J: Cytokine profiles in patients with newly diagnosed multiple myeloma: Survival is associated with IL-6 and IL-17A levels. Cytokine 138: 155358, 2021.

13. Toscani D, Bolzoni M, Ferretti M, Palumbo C and Giuliani N: Role of osteocytes in myeloma bone disease: Anti-sclerostin antibody as new therapeutic strategy. Front Immunol 9: 2467, 2018.

14. Cetin G, Eşkazan AE, Ar MC, Aydın ŞÖ, Ferhanoğlu B, Soysal T, Başlar Z and Aydın Y: Bone-specific alkaline phosphatase levels among patients with multiple myeloma receiving various therapy options. Turk J Haematol 31: 374-380, 2014.

15. Nizet A, Cavalier E, Stenvinkel P, Haarhaus M and Magnusson P. Bone alkaline phosphatase: An important biomarker in chronic kidney disease-mineral and bone disorder. Clin Chim Acta 501: 198-206, 2020.

16. Jasrotia S, Gupta R, Sharma A, Halder A and Kumar L: Cytokine profile in multiple myeloma. Cytokine 136: 155271, 2020.

17. Burger R, Günther A, Klausz K, Staudinger M, Peipp M, Penas EM, Rose-John S, Wijdenes J and Gramatzki M: Due to interleukin-6 type cytokine redundancy only glycoprotein 130 receptor blockade efficiently inhibits myeloma growth. Haematologica 102: 381-390, 2017.

18. Vainer N, Dehlendorff C and Johansen JS: Systematic literature review of IL-6 as a biomarker or treatment target in patients with gastric, bile duct, pancreatic and colorectal cancer. Oncotarget 9: 29820-29841, 2018.

19. Trigo FM, Luizon MR, Dutra HS, Maiolino A, Nucci M and Simões BP: Interaction between IL- 6 and TNF- $\alpha$ genotypes associated with bacteremia in multiple myeloma patients submitted to autologous stem cell transplantation (ASCT). Leuk Res Rep 3: 76-78, 2014.

20. Wu XY, Tian F, Su MH, Wu M, Huang Y, Hu LH, Jin Land Zhu XJ: BF211, a derivative of bufalin, enhances the cytocidal effects in multiple myeloma cells by inhibiting the IL-6/JAK2/STAT3 pathway. Int Immunopharmacol 64: 24-32, 2018.

21. Matthes T, Manfroi B and Huard B: Revisiting IL-6 antagonism in multiple myeloma. Crit Rev Oncol Hematol 105: 1-4, 2016.

22. Ameri Z, Ghiasi S, Farsinejad A, Hassanshahi G, Ehsan M and Fatemi A: Telomerase inhibitor MST-312 induces apoptosis of multiple myeloma cells and down-regulation of anti-apoptotic, proliferative and inflammatory genes. Life Sci 228: 66-71, 2019.

23. Harmer D, Falank C and Reagan MR: Interleukin-6 interweaves the bone marrow microenvironment, bone loss, and multiple myeloma. Front Endocrinol (Lausanne) 9: 788, 2019.

24. Mahdavi Sharif P, Jabbari P, Razi S, Keshavarz-Fathi M and Rezaei N: Importance of TNF-alpha and its alterations in the development of cancers. Cytokine 130: 155066, 2020.

25. Lemancewicz D, Bolkun L, Jablonska E, Kulczynska A, Bolkun-Skornicka U, Kloczko J and Dzieciol J: Evaluation of TNF superfamily molecules in multiple myeloma patients: Correlation with biological and clinical features. Leuk Res 37: 1089-1093, 2013.

26. Kovacs-Benke E: Monitoring disease status in multiple myeloma in view of proliferation-leading cytokines. Cancer Immunol Res 2: 1010, 2017.

27. Li A, Sun K, Wang J, Wang S, Zhao X, Liu R and Lu Y: Recombinant expression, purification and characterization of human soluble tumor necrosis factor receptor 2. Protein Expr Purif 182: 105857, 2021

28. Hong Y, Yu J, Wang G and Qiao W: Association between tumor necrosis factor alpha gene polymorphisms and multiple myeloma risk: An updated meta-analysis. Hematology 24: 216-224, 2019

29. D'Souza C, Prince H and Neeson P: Understanding the role of T-cells in the antimyeloma effect of immunomodulatory drugs. Front Immunol 12: 632399, 2021.

30. Børset M, Sundan A, Waage A and Standal T: Why do myeloma patients have bone disease? A historical perspective. Blood Rev 41: 100646, 2020.

31. Kleber M, Ntanasis-Stathopoulos I, Dimopoulos MA and Terpos E: Monoclonal antibodies against RANKL and sclerostin for myeloma-related bone disease: Can they change the standard of care? Expert Rev Hematol 12: 651-663, 2019.

32. Marino S and Roodman GD: Multiple myeloma and bone: The fatal interaction. Cold Spring Harb Perspect Med 8: a031286, 2018. 
33. Abramson HN: Monoclonal antibodies for the treatment of multiple myeloma: An update. Int J Mol Sci 19: 3924, 2018.

34. Terpos E, Raje N, Croucher P, Garcia-Sanz R, Leleu X, Pasteiner W, Wang Y, Glennane A, Canon J and Pawlyn C: Denosumab compared with zoledronic acid on PFS in multiple myeloma: Exploratory results of an international phase 3 study. Blood Adv 5: 725-736, 2021.

35. Terpos E, Ntanasis-Stathopoulos I and Dimopoulos M: Myeloma bone disease: From biology findings to treatment approaches. Blood 133: 1534-1539, 2019.

36. Chubb SAP and Vasikaran SD: Measurement and clinical utility of $\beta C T X$ in serum and plasma. Adv Clin Chem 81: 97-134, 2017.

37. Shetty S, Kapoor N, Bondu JD, Thomas N and Paul TV: Bone turnover markers: Emerging tool in the management of osteoporosis. Indian J Endocrinol Metab 20: 846-852, 2016.

38. Ting KR, Brady JJ, Hameed A, Le G, Meiller J, Verburgh E, Bayers C, Benjamin D, Anderson KC, Richardson PG, et al: Clinical utility of C-terminal telopeptide of type 1 collagen in multiple myeloma. Br J Haematol 173: 82-88, 2016.

39. Lorentzon M, Branco J, Brandi ML, Bruyère $O$, Chapurlat $R$, Cooper C, Cortet B, Diez-Perez A, Ferrari S, Gasparik A, et al: Algorithm for the use of biochemical markers of bone turnover in the diagnosis, assessment and follow-up of treatment for osteoporosis. Adv Ther 36: 2811-2824, 2019.

40. Vallet S, Hoyle NR, Kyle RA, Podar K and Pecherstorfer M: A role for bone turnover markers $\beta$-CrossLaps (CTX) and amino-terminal propeptide of type I collagen (PINP) as potential indicators for disease progression from MGUS to multiple myeloma. Leuk Lymphoma 59: 2431-2438, 2018.
41. Tian A, Ma J, Feng K, Liu Z, Chen L, Jia H and Ma X: Reference markers of bone turnover for prediction of fracture: A meta-analysis. J Orthop Surg Res 14: 68, 2019.

42. Terpos E, Dimopoulos MA, Sezer O, Roodman D, Abildgaard N, Vescio R, Tosi P, Garcia-Sanz R, Davies F, Chanan-Khan A, et al: The use of biochemical markers of bone remodeling in multiple myeloma: A report of the international myeloma working group. Leukemia 24: 1700-1712, 2010.

43. Raje N, Bhatta S and Terpos E: Role of the RANK/RANKL pathway in multiple myeloma. Clin Cancer Res 25: 12-20, 2019.

44. Maaroufi A, Khadem-Ansari MH, Khalkhali HR and Rasmi Y: Serum levels of bone sialoprotein, osteopontin, and $\beta 2$-microglobulin in stage I of multiple myeloma. J Cancer Res Ther 16: 98-101, 2020.

45. Terpos E: Biochemical markers of bone metabolism in multiple myeloma. Cancer Treat Rev 32 (Suppl 1): S15-S19, 2006.

46. Zhou F, Meng S, Song H and Claret FX: Dickkopf-1 is a key regulator of myeloma bone disease: Opportunities and challenges for therapeutic intervention. Blood Rev 27: 261-267, 2013. 